\title{
The Use of Microwave Technology in a Clinical E.M. Laboratory
}

\author{
R. L. Austin \\ Louisiana State University Medical Center, Dept. of Pathology, Electron Microscopy \\ Laboratory, Shreveport, LA. 71130
}

Since the first modified microwave oven, to process tissue for E.M., was marketed by Ted Pella in 1994, the microwave has advanced into a more sophisticated instrument allowing for even faster processing time than the present literature has suggested [1]. In a previous publication by this author clinical specimens were processed with an average processing time of about 4 hours from wet tissue to polymerized block, with another 1 to $1 \frac{1}{2}$ hours to get the tissue to the E.M. for diagnostic viewing (Fig. 1b). This produced a processing time of about 5 to 6 hours depending on the number of specimens to be processed, sectioned, and stained. At that time this was a great improvement in turn around time from previous methods. The microwave of just a few years ago had a fixed wattage setting of approximately 750 watts, and the bad cooler temperature was set at a fixed $40^{\circ} \mathrm{C}$ before it would start cooling the circulating water load (Fig. 1a). The additional water loads had to be manually reloaded and readjusted to eliminate hot spots (Fig.1a). Specimens and fixative had to be pre-cooled in ice baths before they could be safely microwaved [2].

Microwave processing has been simplified through the development of a water re circulation device called the ColdSpot ${ }^{\mathrm{TM}}$. This device brings about the virtual elimination of hot and cold spots in the microwave cavity. It thus provides a uniform surface area (8.5"x11.0") to process tissue, eliminating the need for ice baths and additional water loads. The load cooler has an adjustable temperature control so that a temperature lower than $40^{\circ} \mathrm{C}$ can be maintained in the ColdSpot ${ }^{\mathrm{TM}}$ cooler processing conditions. The wattage setting is now adjustable from a fixed 750 watts in the old Model 3450 to as low as 150 watts in the Model 3451 and lower still in the newest microwave device with the incorporation of a wattage control mechanism. In addition, a vacuum system can be easily used in combination with the ColdSpot ${ }^{\mathrm{TM}}$. These features, in combination with the variable temperature load cooler, (Fig. 2a) enhance fixation and infiltration and reduce the processing time even further [3]. We are now looking at a processing time of 2 hours from wet to polymerized tissue. This is twice as fast as the earlier microwave capabilities. Considering an added 1 to $1 \frac{1}{2}$ hours for sectioning and staining we now have a system that can get newly received tissue to the electron microscope for diagnostic viewing in roughly 3 to $3 \frac{1}{2}$ hours (Fig. 2b).

\section{References}

[1] R.T. Giberson et al., J. Vet. Diagn. Invest 9 (1997) 61.

[2] R. L. Austin., Microwave, Techniques and Protocols. Humana Press Inc. (2001) 38.

[3] Ted Pella et al., Pelco Inc. Technical Notes. (2001) 1. 


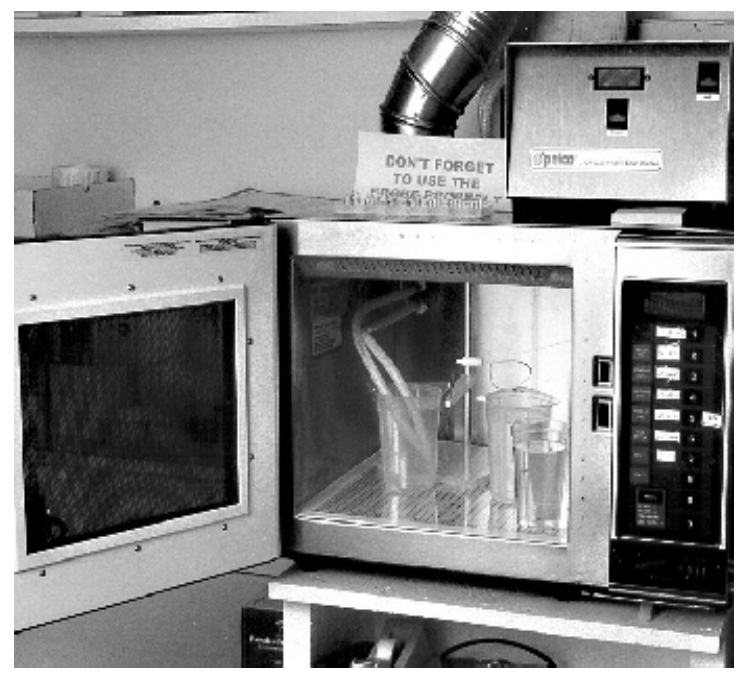

Figure 1a. Original microwave model 3450 series without wattage control or cold spot.

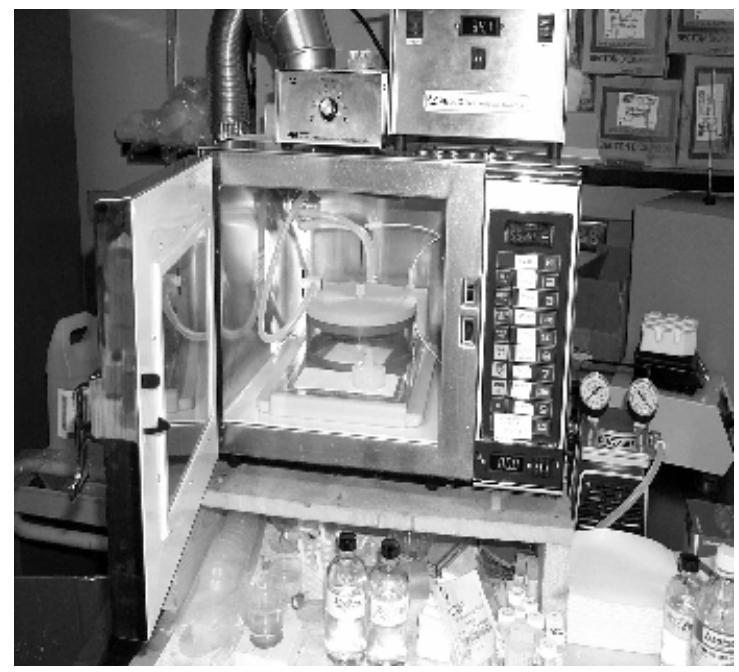

Figure 2a. The newer version of the model 3450 series show variable wattage control unit and cold spot, with vacuum chamber in place.

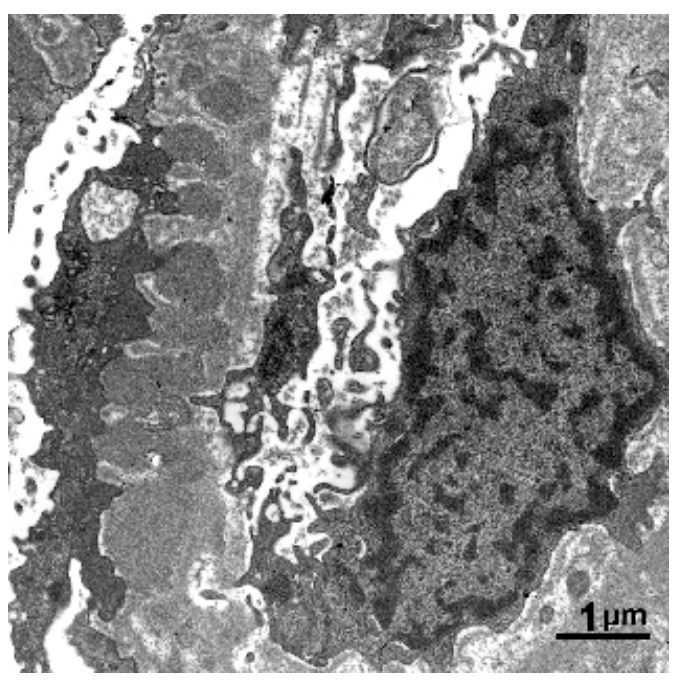

Figure 1b. Results

demonstrate oven capabilities

to process tissue.

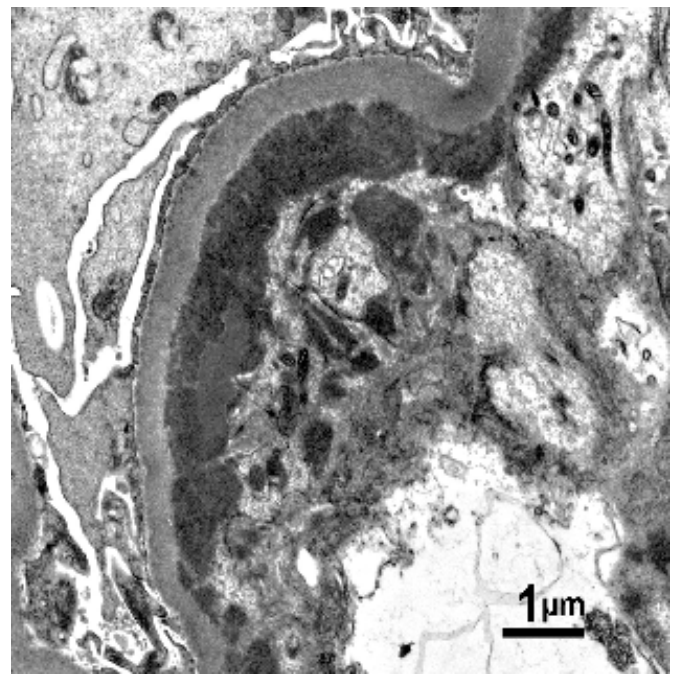

Figure 2b. Results from the improvements made to the microwave oven. 